\title{
The possible association of steroids with fluid accumulation in critically ill patients - a case of a potential bias
}

Amit Frenkel ( $\sim$ frenkela@clalit.org.il )

Soroka Medical Center https://orcid.org/0000-0002-4162-8406

\section{Ran Abuhasira}

Soroka Medical Center

\section{Yoav Bichovsky}

Soroka Medical Center

\section{Anton Bukhin}

Ben-Gurion University of the Negev

Victor Novack

Soroka Medical Center

\section{Evgeni Brotfain}

Soroka Medical Center

\section{Alexander Zlotnik \\ Soroka Medical Center \\ Moti Klein \\ Soroka Medical Center}

\section{Research}

Keywords: Glucocorticoids, Steroids, Fluid, Balance, Sepsis, Septic Shock

Posted Date: March 5th, 2020

DOl: https://doi.org/10.21203/rs.3.rs-16269/v1

License: (1) This work is licensed under a Creative Commons Attribution 4.0 International License. Read Full License 

critically ill patients - a case of a potential bias

4 Amit Frenkel MD MHA ${ }^{\mathrm{a}^{*}}$, Ran Abuhasira MD ${ }^{\mathrm{b}^{*}}$, Yoav Bichovsky MD ${ }^{\mathrm{a}}$, Anton

$5 \quad$ Bukhin B.Med.Sc ${ }^{\mathrm{c}}$, Victor Novack MD PhD ${ }^{\mathrm{b}, \mathrm{d}}$, Evgeni Brotfain $\mathrm{MD}^{\mathrm{a}}$, Alexander

6 Zlotnik $M D P h D^{\mathrm{e}}$, Moti Klein $M D^{\mathrm{a}}$

$8{ }^{\mathrm{a}}$ General Intensive Care Unit, Soroka University Medical Center, and The Faculty of

9 Health Sciences, Ben-Gurion University of the Negev, Beer-Sheva, Israel

$10{ }^{\mathrm{b}}$ Clinical Research Center, Soroka University Medical Center, and The Faculty of

11 Health Sciences, Ben-Gurion University of the Negev, Beer-Sheva, Israel

$12{ }^{c}$ The Joyce and Irving Goldman Medical School, Faculty of Health Sciences, Ben-

13 Gurion University of the Negev, Beer-Sheva, Israel

$14{ }^{\mathrm{d}}$ Anesthesia, Critical Care and Pain Medicine, Beth Israel Deaconess Medical Center,

15 Harvard Medical School, Boston, USA

$16{ }^{\mathrm{e}}$ Department of Anesthesiology, Soroka University Medical Center, and The Faculty

17 of Health Sciences, Ben-Gurion University of the Negev, Beer-Sheva, Israel

$18 *$ equal contribution

19 Corresponding author:

20 Dr. Amit Frenkel, M.D, M.H.A

21 P.O. Box 151, Beer-Sheva 84101, Israel

22 Tel. +972-8-640-0727, Fax: +972-8-640-3390, e-mail: frenkela@ clalit.org.il 


\section{Abstract}

Background: Glucocorticoids (GCS) are commonly administered to critically ill patients. Due to their mineralocorticoid effect, GCS might have a substantial influence on a positive fluid balance. We assessed the association between glucocorticoids (GCS) therapy and fluid balance in critically ill patients with sepsis.

Methods: This is a retrospective study of patients with sepsis hospitalized during 2006-2018 in a general intensive care unit (ICU) at a 1100-bed tertiary medical center.

Results - We considered two definitions of exposure: daily exposure to GCS and GCS treatment at any time in the ICU. Of 945 patients with a diagnosis of sepsis, 375 were treated with GCS at any time and 570 were not. We applied four regression models to assess the association between GCS treatment and fluid balance; in our first model, fluid balance did not differ during days with GCS treatment, between patients who were and were not treated with GCS in the ICU (coefficient estimate $79.5(-55.4$ to 214.4$), p=0.25)$. In our second model, daily fluid balance was increased by 139.8 $\mathrm{ml}(10.8$ to $268.9 ; \mathrm{p}=0.03)$ in patients who were ever treated with GCS during their ICU stay compared to untreated patients. In the third model, which included only patients treated with GCS during their ICU stay, GCS treatment days were not associated with daily fluid balance (coefficient estimate -190.6 (-485.1 to 103.9), pvalue $=0.21$ ). In the last model, on "steroid free days", patients who received GCS treatment during their ICU stay had a positive fluid balance compared to those who were never treated with steroids (coefficient estimate 157.7 (-24.6 to 340.1), pvalue $=0.09$ ). 
49 Conclusions - Despite their known mineralocorticoid activity, GCS themselves

50 appear not to contribute substantially to fluid retention. The findings highlight the

51 importance of a clear definition of exposure.

52 Keywords: Glucocorticoids; Steroids; Fluid; Balance; Sepsis; Septic Shock 


\section{Introduction}

A growing body of evidence suggests that a positive fluid balance in patients hospitalized in intensive care is directly related to worse outcomes [1,2], and is an independent negative prognostic factor in patients with sepsis [1,3]. Glucocorticoids (GCS) are commonly administered to critically ill patients for a wide range of indications, especially septic shock [4]. GCS might have a substantial influence on a positive fluid balance, mostly due to their mineralocorticoid effect. Increased mineralocorticoid activity and high aldosterone levels cause an increase in sodium reabsorption in the renal tubule [5]. Additionally, some evidence suggests that GCS may overcome the effect of decreased expression of the mineralocorticoid receptor, due to increased levels of tumor necrosis factor- $\alpha$ in critical care patients [6], thus contributing to fluid retention [7].

According to the "Surviving Sepsis Campaign" Guidelines for Management of Sepsis and Septic Shock [8], GCS are recommended for septic shock that is refractory to adequate fluid resuscitation and vasopressor administration. Consequently, patients with sepsis who are treated with GCS are presumably with a more severe disease than patients not treated with GCS. Thus, a possible association between GCS use and fluid balance could be due to the indication for GCS treatment rather than the GCS treatment itself. This raises a core question in the interpretation of observational studies: is the association observed due to a true effect of the exposure or rather the result of a bias?

We conducted a retrospective study to assess the association between GCS therapy and fluid balance in critically ill patients with sepsis. To examine whether a possible association might be due to an indication bias, we analyzed the data according to two definitions of exposure: per GCS treatment at any time during 
intensive care unit (ICU) stay and GCS treatment per day of stay. In the analysis that used the latter definition, the control group included patients without any GCS treatment and also "steroid-free days" of patients treated with GCS during their ICU stay. We hypothesized that analyses using the two definitions of GCS treatment would yield different results.

\section{Materials and Methods}

\section{Study design and study population}

We conducted a population-based retrospective cohort study at Soroka University Medical Center, a tertiary care medical center that serves as the only regional hospital in southern Israel (Beer-Sheva vicinity, estimated population of $1,000,000)$. We included all adult patients hospitalized with a diagnosis of sepsis in the general ICU for 24 hours or more, between December 2006 and January 2018. We identified patients with sepsis according to either the International Classification of Diseases, 9th revision (ICD-9) codes or a diagnosis in the internal ICU medical record. Comorbidities were also defined by ICD-9 codes. We used the Sequential Organ Failure Assessment (SOFA) score to evaluate organ dysfunction at ICU admission [9]. Exclusion criteria were hemofiltration or dialysis treatment during the ICU stay.

\section{Clinical definitions and data sources}

GCS treatment was defined as the administration of one of the following drugs, at least once, during the ICU stay: hydrocortisone, methylprednisolone, or prednisone. Indications for steroid treatment were septic shock or acute respiratory distress syndrome.

Using the first definition of GCS exposure, we compared patients who did and did not receive GCS therapy during their ICU stay. Using the second definition of 
103 GCS exposure, we aggregated patients' data to 24-hour periods. For each of these

104 periods, we analyzed the use of steroids, the daily fluid balance, and serum creatinine

105 for each patient. The daily fluid balance was calculated as the total daily input

106 (nutrition, crystalloids, blood products, intravenous drugs) minus the total daily output

107 (urine, fluids from body drains), and presented in milliliter units. We evaluated the

108 creatinine level for every admission day. We used the average daily value when a

109 particular test was performed more than once in a single day. Days in which patients

110 were treated with diuretics were excluded from the analysis. For patients who had

111 more than one admission in the ICU during the study period, we used only the first

112 admission. We limited the analysis to the first 21 ICU hospitalization days. For both

113 definitions of exposure, the outcome was the daily fluid balance during the ICU

114 admission.

115 Statistical analysis

116 The results are presented by means \pm SDs for continuous variables, medians

117 and interquartile ranges for ordinal variables, and percentages for categorical data.

118 The Chi-square test was used for categorical variables, the t-test for continuous

119 variables, and the Mann-Whitney test for ordinal variables.

120 Linear generalized estimating equation (GEE) models with unstructured

121 correlation matrices were used to estimate associations between steroid treatment on

122 each day of admission and the daily fluid balance. GEE models were used to account

123 for repeated measurements of fluid balance in the same patient. In all the models, the

124 dependent variable was the total daily fluid balance in milliliters. The independent

125 variables were defined a priori as fixed effects; firstly, steroid treatment per day or at

126 any time during the ICU stay, depending on the approach used. In addition, the 
127 following variables were considered: age, sex, SOFA score at admission, average

128 serum creatinine level, and admission day number in the ICU.

We applied four models to determine the contribution of steroid treatment to

130 the fluid balance (Figures 1-4). In the first model, we compared the fluid balance

131 between all the days with GCS treatment and all the days without GCS. The latter was

132 derived from two sub-groups: the first, "steroid-free days" in patients receiving GCS

133 treatment during their ICU stay; and the second, all ICU days of patients who were

134 not treated with GCS. In the second model, we compared all the days of patients who

135 were not treated with GCS during their ICU stay to all the days of patients who

136 received GCS treatment during their ICU stay. In the third model, we included only

137 the patients who were treated with GCS during their ICU stay. We compared days

138 with GCS treatment to days without GCS treatment. In the last model, we compared

139 all the days of patients who were never treated with GCS during their ICU stay to

140 "steroid-free days" of those who received GCS treatment at some time during their

141 ICU stay. SPSS IBM software, version 25.0, was used for statistical analysis.

142 Data availability

143 The data used in the analysis of this study are not publicly available due to the

144 national regulations but are available from the corresponding author upon request.

146 Results

147 Study Population

148 The study included 945 patients: 375 (39.7\%) who were treated with GCS

149 during their ICU stay and 570 who were not. Table 1 summarizes the characteristics

150 of the study population: the mean age was lower among those treated than not treated

151 with GCS $(57.5 \pm 21.3$ vs. $61.2 \pm 18.9, \mathrm{p}=0.006)$. The majority of the patients in both 
groups were males (61.6\% and 59.5\%, respectively). The admission SOFA Score (median, interquartile range (IQR)) was higher among those treated than not treated with GCS (11 (9-13)) vs. $(10(7-12)), \mathrm{p}<0.001$. Patients with a history of chronic obstructive pulmonary disease constituted $6.1 \%$ of those treated with GCS and $1.9 \%$ of those not treated $(\mathrm{p}<0.001)$. The duration of hospitalization (median, IQR) and the mortality rate in the ICU were higher among those treated than not treated with GCS (10 (3-26) days) vs. (4 (1-14) days) and $25.6 \%$ vs. $14.6 \%$, respectively.

\section{Steroid treatment and fluid balance}

The first model compared fluid balance between all the days with GCS treatment and all the days without GCS. Female sex, older age, higher SOFA score on admission, and high creatinine were all shown to be associated with significantly increased daily fluid balance (Figure 1). This model demonstrated no significant association of GCS treatment with daily fluid balance (coefficient estimate 79.5 (-55.4 to 214.4), p-value=0.25).

The second model compared all the days hospitalized in the ICU, between patients not treated with GCS and patients who received GCS treatment. All the variables mentioned above were associated with significantly increased daily fluid balance (Figure 2). However, the daily fluid balance was increased in patients treated than not treated with GCS, by $139.8 \mathrm{ml}(10.8$ to $268.9 ; \mathrm{p}=0.03)$.

The third model included only patients treated with GCS during their ICU stay and compared "steroid-free days" to days with GCS treatment. We found no significant association of GCS treatment with daily fluid balance (coefficient estimate $-190.6(-485.1$ to 103.9$), \mathrm{p}$-value=0.21) $($ Figure 3).

In the last model, patients who received GCS treatment during their ICU stay had a positive fluid balance on their "steroid-free days" compared to those who were 
178 (Figure 4).

179

\section{Discussion}

Our analysis of data according to two definitions of GCS exposure yielded different results, thus confirming our hypothesis. Specifically, in our cohort of patients with sepsis, we found an association of GCS therapy with positive fluid balance when the exposure was defined as any treatment with GCS during the ICU stay. However, when the exposure was defined as daily exposure to GCS, this association did not hold. Thus, this observational study suggests that evidence of an association between GCS therapy and fluid balance is due to an indication bias, and not to a true effect of GCS treatment. The bias stems from the greater disease severity of the patients treated with GCS. In further support of this interpretation, a positive fluid balance was observed on "steroid-free days" in patients treated with GCS compared to patients not treated with GCS during their ICU stay.

Steroids are widely used in patients with septic shock, while survival benefit has been shown only among those who remained hypotensive after fluid and vasopressor resuscitation [10]. The rationale for glucocorticoid administration in this population is based on data suggesting that critical illness induces a relative adrenal insufficiency that may contribute to shock. Previous studies identified positive fluid balance as a predictor of clinical outcome [2,11]. Moreover, some authors support restrictive intravenous fluid therapy in patients with sepsis to avoid edema within vital organs and organ dysfunction, with impairment of oxygen delivery [11]. In this study, we applied four models to determine the contribution of steroids treatment to fluid balance in patients hospitalized in the ICU with sepsis. Our first 
model examined GCS per treatment days. Days with GCS treatment were compared to days without GCS treatment, between patients who were treated with GCS at any time during their ICU stay and those who were not treated with GCS during their ICU stay. This model showed no association of GCS treatment with daily fluid balance. However, in the second model, we compared between patients who received GCS

207 treatment at least once to those who did not receive GCS treatment during their ICU 208 stay. GCS treatment was shown to be associated with a positive daily fluid balance.

209 The conclusion from these two models is that the steroids themselves do not influence 210 the daily fluid balance. Rather, the propensity to treat the more severe patients with

211 GCS may explain the positive balance in those patients. This hypothesis is supported 212 by the third model, which assessed only patients who received GCS treatment. Here, 213 no difference was observed in daily fluid balance between the days with and without 214 GCS treatment. The last model compared only days without GCS treatment, between 215 patients who were and were not treated with GCS. Patients who received GCS 216 treatment at any time during their ICU admission demonstrated positive fluid balance. 217 This again shows that the clinical characteristics leading to steroid treatment rather 218 than steroids themselves are responsible for the fluid retention.

219 The severity of disease in the patients treated with GCS is evidenced by their 220 older age, higher SOFA scores, longer admission times, higher ventilation rates, and 221 higher mortality rates. This is consistent with the indications for GCS according to the 222 guidelines for patients with septic shock $[8,10]$. The differences in characteristics 223 between the study groups support our supposition that the association between GCS 224 treatment and a positive fluid balance stems from an indication bias, whereby patients 225 with more severe illness and a positive fluid balance are treated with steroids. 
The strengths of our study include a lengthened period of data collection with

227 a relatively large cohort. Yet, our study has a number of limitations. First, it is a

228 retrospective study that included patients with sepsis from a single center. Second, we

229 analyzed associations of overall GCS therapy with fluid balance, while the different

230 steroids that were used to treat our patients (hydrocortisone, methylprednisolone) have

231 unequal mineralocorticoid activity [3].

\section{Conclusions}

233 We suggest that GCS themselves, though known to have mineralocorticoid

234 activity, do not contribute substantially to fluid retention in critically ill patients. This

235 finding may help elucidate the "fluid balance" concept in critically ill patients with

236 sepsis who receive GCS and reduce the concern that GCS causes a positive fluid

237 balance in this population.

\section{List of abbreviations}

240 GCS, glucocorticoids; ICU, intensive care unit; SOFA, Sequential Organ Failure

241 Assessment; GEE, generalized estimating equation; IQR, interquartile range

\section{Declarations}

\section{Ethics approval and consent to participate}

245 The study was approved by the Soroka University Medical Center Ethics Committee.

246 All clinical investigations were conducted according to the principles expressed in the

247 Declaration of Helsinki. The Ethics Committee approval exempted the study from

248 informed consent due to the retrospective data collection that maintained subject

249 confidentiality. Patient records were anonymized and de-identified prior to analysis. 
Consent for publication

251 Not applicable

252 Availability of data and materials

253 The datasets used and/or analysed during the current study are available from the

254 corresponding author on reasonable request.

255 Competing interests

256 The authors declare that they have no competing interests.

257 Funding

258 No external funding was used for this work.

259

260 Authors' contributions

261 AF Review \& Editing

262 RA Review \& Editing

263 YB Methodology and Formal Analysis

264 AB Methodology and Formal Analysis

265 VN Conceptualization

266 EB Validation

267 AZ Writing - Original Draft Preparation

268 MK Supervision

269 All the authors read and approved the final manuscript. 


\section{$\underline{\text { References }}$}

271 1. Acheampong A, Vincent JL. A positive fluid balance is an independent prognostic 272 factor in patients with sepsis. Crit Care [Internet]. BioMed Central Ltd.; 2015 [cited 2732020 Feb 9];19:251. Available from: http://ccforum.com/content/19/1/251

274 2. Brotfain E, Koyfman L, Toledano R, Borer A, Fucs L, Galante O, et al. Positive 275 fluid balance as a major predictor of clinical outcome of patients with sepsis/septic 276 shock after ICU discharge. Am J Emerg Med. W.B. Saunders; 2016;34:2122-6.

277 3. Sakr Y, Rubatto Birri PN, Kotfis K, Nanchal R, Shah B, Kluge S, et al. Higher 278 Fluid Balance Increases the Risk of Death from Sepsis: Results from a Large 279 International Audit* . Crit Care Med. Lippincott Williams and Wilkins; 2017;45:38628094

281 4. Annane D, Renault A, Brun-Buisson C, Megarbane B, Quenot JP, Siami S, et al. 282 Hydrocortisone plus fludrocortisone for adults with septic shock. N Engl J Med 283 [Internet]. Massachussetts Medical Society; 2018 [cited 2020 Feb 9];378:809-18. 284 Available from: http://www.nejm.org/doi/10.1056/NEJMoa1705716

285 5. Epstein M. Aldosterone as a determinant of cardiovascular and renal dysfunction. J. 286 R. Soc. Med. Royal Society of Medicine Press Ltd; 2001. p. 378-83.

287 6. Ramseyer VD, Garvin JL. Tumor necrosis factor- $\alpha$ : Regulation of renal function 288 and blood pressure. Am. J. Physiol. - Ren. Physiol. American Physiological Society; 289 2013. p. 1231-42.

290 7. Fadel F, André-Grégoire G, Gravez B, Bauvois B, Bouchet S, Sierra-Ramos C, et 291 al. Aldosterone and Vascular Mineralocorticoid Receptors in Murine Endotoxic and 292 Human Septic Shock. Crit Care Med. Lippincott Williams and Wilkins; $293 \quad 2017 ; 45: \mathrm{e} 954-62$.

294 8. Rhodes A, Evans LE, Alhazzani W, Levy MM, Antonelli M, Ferrer R, et al. 
295 Surviving Sepsis Campaign: International Guidelines for Management of Sepsis and

296 Septic Shock: 2016. Intensive Care Med. Springer Verlag; 2017;43:304-77.

297 9. Vincent JL, Moreno R, Takala J, Willatts S, De Mendonça A, Bruining H, et al.

298 The SOFA (Sepsis-related Organ Failure Assessment) score to describe organ

299 dysfunction/failure. On behalf of the Working Group on Sepsis-Related Problems of

300 the European Society of Intensive Care Medicine. Intensive Care Med [Internet].

301 Springer-Verlag; 1996 [cited 2019 Apr 15];22:707-10. Available from:

302 https://link.springer.com/content/pdf/10.1007\%2FBF01709751.pdf

303 10. Sprung CL, Annane D, Keh D, Moreno R, Singer M, Freivogel K, et al.

304 Hydrocortisone therapy for patients with septic shock. N Engl J Med [Internet].

305 Massachussetts Medical Society; 2008 [cited 2020 Feb 9];358:111-24. Available

306 from: http://www.nejm.org/doi/abs/10.1056/NEJMoa071366

307 11. Chao WC, Tseng CH, Chien YC, Sheu CC, Tsai MJ, Fang WF, et al. Association

308 of day 4 cumulative fluid balance with mortality in critically ill patients with

309 influenza: A multicenter retrospective cohort study in Taiwan. PLoS One. Public

310 Library of Science; 2018. 
Table 1 - Characteristics of patients at admission to the intensive care unit.

\begin{tabular}{|c|c|c|c|}
\hline Variable & $\begin{array}{l}\text { Treated with steroids } \\
\text { during their ICU stay } \\
\qquad(\mathrm{N}=375)\end{array}$ & $\begin{array}{l}\text { Not treated with } \\
\text { steroids during } \\
\text { their ICU stay } \\
\quad(\mathrm{N}=\mathbf{5 7 0})\end{array}$ & P-value \\
\hline Age (Mean \pm SD) & $61.2 \pm 18.9$ & $57.5 \pm 21.3$ & 0.006 \\
\hline Male $(n, \%)$ & $223(59.5 \%)$ & $351(61.6 \%)$ & 0.52 \\
\hline \multicolumn{4}{|l|}{ Comorbidities (n, \%) } \\
\hline CAD & $74(19.7 \%)$ & $134(23.5 \%)$ & 0.17 \\
\hline COPD & $23(6.1 \%)$ & $11(1.9 \%)$ & 0.001 \\
\hline HF & $39(10.4 \%)$ & $63(11.1 \%)$ & 0.75 \\
\hline HTN & $189(50.4 \%)$ & $263(46.1 \%)$ & 0.20 \\
\hline Cancer & $89(23.7 \%)$ & $103(18.1 \%)$ & 0.03 \\
\hline DM & $115(30.7 \%)$ & $194(34.0 \%)$ & 0.28 \\
\hline PVD & $20(5.3 \%)$ & $42(7.4 \%)$ & 0.22 \\
\hline SOFA Score (Median, IQR) & $11(9-13)$ & $10(7-12)$ & $<0.001$ \\
\hline Cardiovascular sub-score & $4(4-4)$ & $3(2-4)$ & $<0.001$ \\
\hline Renal sub-score & $0(0-1)$ & $0(0-1)$ & $<0.001$ \\
\hline Coagulation sub-score & $0(0-1)$ & $0(0-1)$ & 0.89 \\
\hline Respiration sub-score & $3(2-4)$ & $3(2-4)$ & 0.003 \\
\hline Liver sub-score & $0(0-1)$ & $0(0-1)$ & 0.37 \\
\hline CNS sub-score & $4(2-4)$ & $4(0-4)$ & 0.003 \\
\hline GCS treatment days (Median, IQR) & $3(2-10)$ & - & - \\
\hline In-ICU death $(\mathrm{n}, \%)$ & $96(25.6 \%)$ & $83(14.6 \%)$ & $<0.001$ \\
\hline ICU admission days & $10(3-26)$ & $4(1-14)$ & $<0.001$ \\
\hline Mechanically ventilated (n, \%) & $354(94.4 \%)$ & $440(77.2 \%)$ & $<0.001$ \\
\hline
\end{tabular}

315 ICU - Intensive Care Unit; CAD - Coronary Artery Disease; COPD - Chronic Obstructive

316 Pulmonary Disease; HF - Heart Failure; HTN - Hypertension; DM - Diabetes Mellitus; PVD

317 - Peripheral Vascular Disease; SOFA - Sequential Organ Failure Assessment; GCS -

318 glucocorticoids; CNS - central nervous system. 
Figure 1 - Model 1 of the estimated effect of glucocorticoids (GCS) treatment per day on daily fluid balance (ml) in patients with sepsis in the intensive care unit (ICU). In this model, all the days with GCS were compared to all the days without GCS.

Figure 2 - Model 2 of the estimated effect of glucocorticoids (GCS) treatment per day on daily fluid balance (ml) in patients with sepsis in the intensive care unit (ICU). In this model, all the hospitalized days of patients who were treated with GCS were compared to all the hospitalized days of patients who were not treated with GCS.

Figure 3 - Model 3 of the estimated effect of glucocorticoids (GCS) treatment per day on daily fluid balance $(\mathrm{ml})$ in patients with sepsis who were treated with GCS during their stay in the intensive care unit (ICU). This model included only patients treated with GCS during their ICU stay and compared between GCS days and GCSfree days.

$\underline{\text { Figure } 4}$ - Model 4 of the estimated effect of ever being treated with glucocorticoids (GCS) on daily fluid balance (ml) in patients with sepsis in the intensive care unit. 
Figures

\begin{tabular}{|l|c|c|}
\hline \multicolumn{1}{|c|}{ Variable } & $\begin{array}{c}\text { Coefficient estimate (ml) } \\
(\mathbf{9 5 \%} \text { CI) }\end{array}$ & p-value \\
\hline Male vs. Female & $-291.5(-423.9$ to -159.2$)$ & $<\mathbf{0 . 0 0 1}$ \\
\hline GCS treatment days & $79.5(-55.4$ to 214.4$)$ & 0.25 \\
\hline Age (for every added year) & $12.1(9.1$ to 15.2$)$ & $<\mathbf{0 . 0 0 1}$ \\
\hline SOFA score (for every added point) & $51.9(30.6$ to 73.1$)$ & $<\mathbf{0 . 0 0 1}$ \\
\hline Creatinine (for every rise in $\mathbf{1} \mathbf{~ m g / d L )}$ & $126.7(41.7$ to 211.7$)$ & $\mathbf{0 . 0 0 3}$ \\
\hline
\end{tabular}

SOFA - Sequential Organ Failure Assessment.

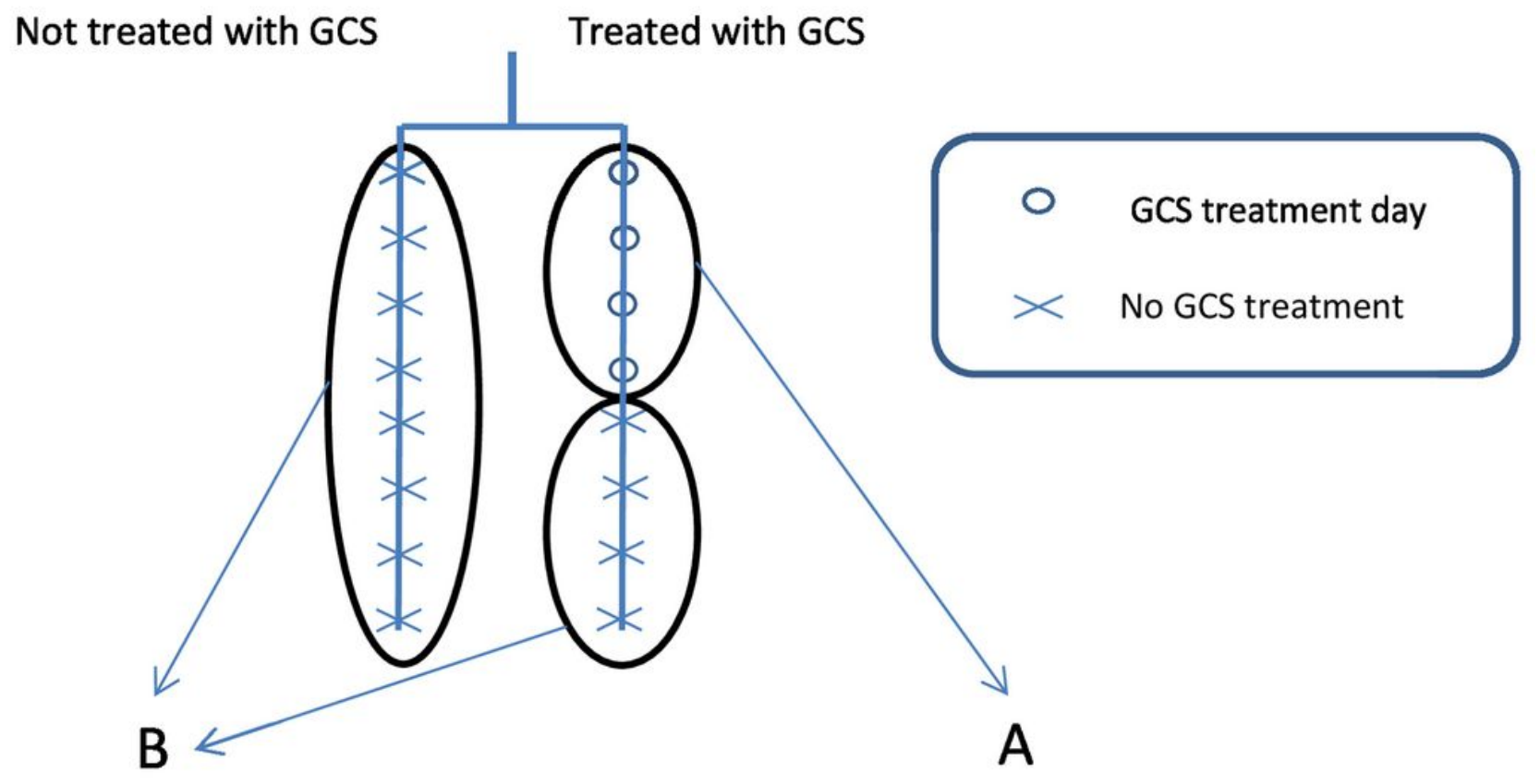
A - Days with GCS treatment
B - Days without GCS treatment in patients treated with GCS, and days in the ICU of patients not treated with GCS

Figure 1

Model 1 of the estimated effect of glucocorticoids (GCS) treatment per day on daily fluid balance (ml) in patients with sepsis in the intensive care unit (ICU). In this model, all the days with GCS were compared to all the days without GCS. 


\begin{tabular}{|l|c|c|}
\hline \multicolumn{1}{|c|}{ Variable } & $\begin{array}{c}\text { Coefficient estimate (ml) } \\
\text { (95\% CI) }\end{array}$ & p-value \\
\hline Male vs. Female & $-289.7(-422.2$ to -157.2$)$ & $<\mathbf{0 . 0 0 1}$ \\
\hline GCS treatment ever & $139.8(10.8$ to 268.9$)$ & $\mathbf{0 . 0 3}$ \\
\hline Age (for every added year) & $12(8.9$ to 15.1$)$ & $<\mathbf{0 . 0 0 1}$ \\
\hline SOFA score (for every added point) & $49.4(28.2$ to 70.5$)$ & $<\mathbf{0 . 0 0 1}$ \\
\hline Creatinine (for every rise in 1 mg/dL) & $129.6(44.8$ to 214.4$)$ & $\mathbf{0 . 0 0 3}$ \\
\hline
\end{tabular}

ICU - Intensive Care Unit; SOFA - Sequential Organ Failure Assessment.

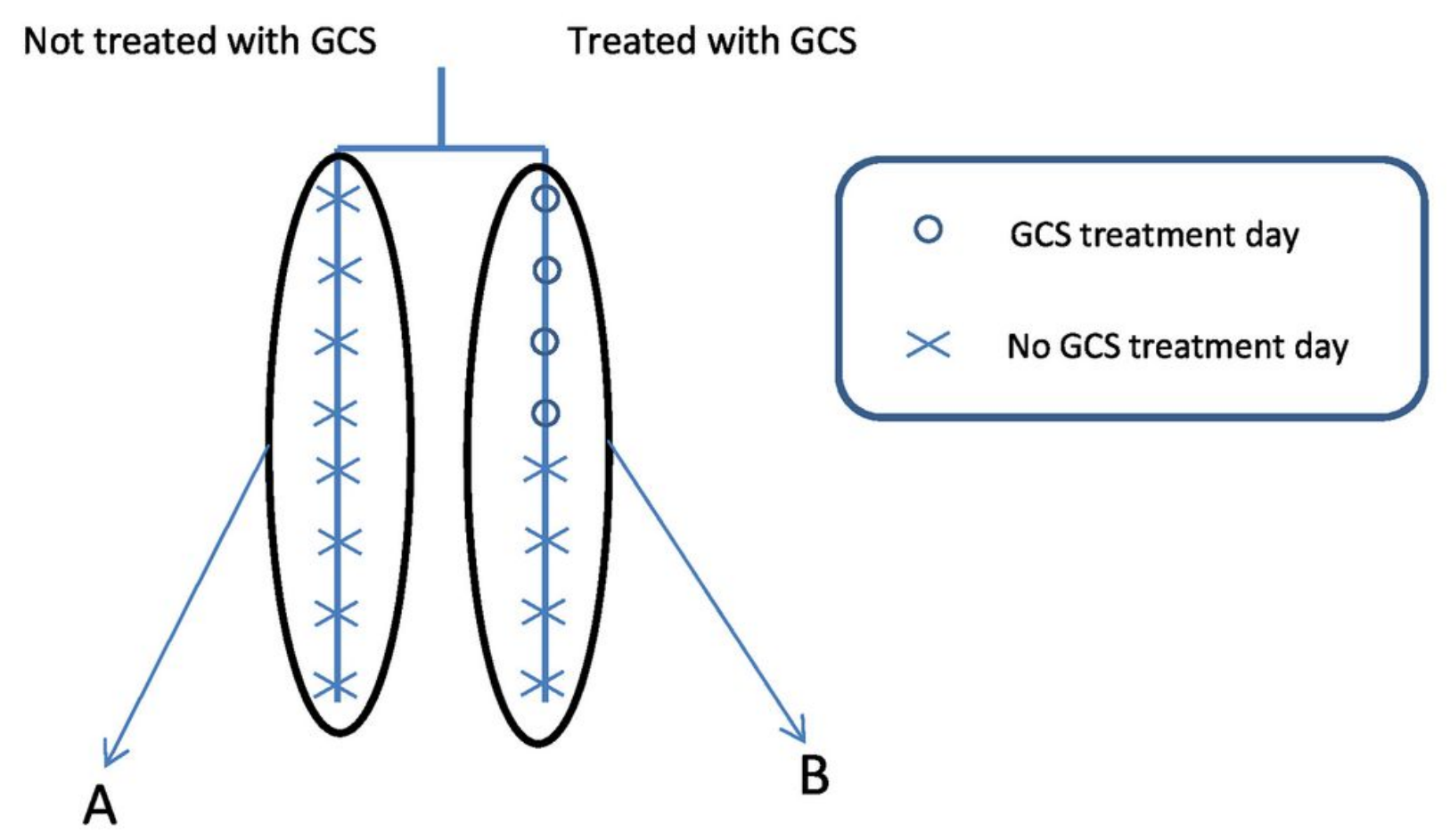

A - All the days of patients who were not treated with GCS

B - All the days of patients who were treated with GCS

Figure 2

Model 2 of the estimated effect of glucocorticoids (GCS) treatment per day on daily fluid balance $(\mathrm{ml})$ in patients with sepsis in the intensive care unit (ICU). In this model, all the hospitalized days of patients who were treated with GCS were compared to all the hospitalized days of patients who were not treated with GCS. 


\begin{tabular}{|l|c|c|}
\hline \multicolumn{1}{|c|}{ Variable } & $\begin{array}{c}\text { Coefficient estimate (ml) } \\
\text { (95\% CI) }\end{array}$ & p-value \\
\hline Male vs. Female & $-272.9(-476.4$ to -69.3$)$ & $\mathbf{0 . 0 1}$ \\
\hline Days with GCS treatment & $-190.6(-485.1$ to 103.9$)$ & 0.21 \\
\hline Age (for every added year) & $12.4(7.3$ to 17.6$)$ & $<\mathbf{0 . 0 0 1}$ \\
\hline SOFA score (for every added point) & $39.2(1.7$ to 76.7$)$ & $\mathbf{0 . 0 4}$ \\
\hline Creatinine (for every rise in $\mathbf{1} \mathbf{~ m g / d L )}$ & $180.4(41.3$ to 319.5$)$ & $\mathbf{0 . 0 1}$ \\
\hline
\end{tabular}

SOFA - Sequential Organ Failure Assessment.

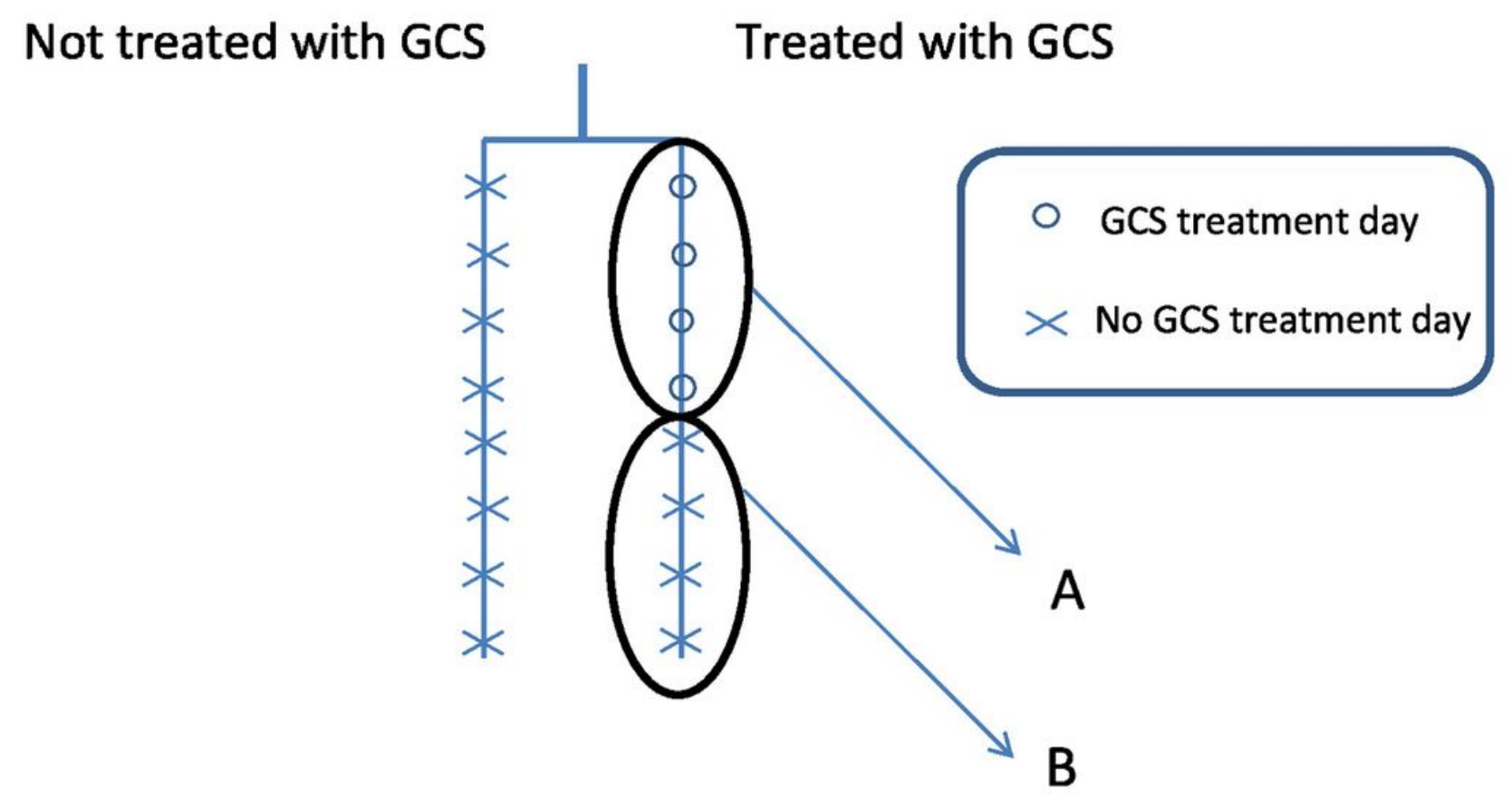

A - Days with GCS treatment

B - "GCS-free days", in patients who were ever treated with GCS during their hospital stay.

Figure 3

Model 3 of the estimated effect of glucocorticoids (GCS) treatment per day on daily fluid balance $(\mathrm{ml})$ in patients with sepsis who were treated with GCS during their stay in the intensive care unit (ICU). This model included only patients treated with GCS during their ICU stay and compared between GCS days and GCS-free days. 


\begin{tabular}{|l|c|c|}
\hline \multicolumn{1}{|c|}{ Variable } & $\begin{array}{c}\text { Coefficient estimate (ml) } \\
\text { (95\% CI) }\end{array}$ & p-value \\
\hline Male vs. Female & $-250.5(-420.2$ to -80.8$)$ & $\mathbf{0 . 0 0 4}$ \\
\hline GCS treatment ever & $157.7(-24.6$ to 340.1$)$ & $\mathbf{0 . 0 9}$ \\
\hline Age (for every added year) & $12.2(8$ to 16.5$)$ & $<\mathbf{0 . 0 0 1}$ \\
\hline SOFA score (for every added point) & $35.5(5.4$ to 65.6$)$ & $\mathbf{0 . 0 2}$ \\
\hline Creatinine (for every rise in 1 $\mathbf{~ m g / d L ) ~}$ & $100.1(-26.4$ to 226.7$)$ & $\mathbf{0 . 1 2 1}$ \\
\hline
\end{tabular}

SOFA - Sequential Organ Failure Assessment.

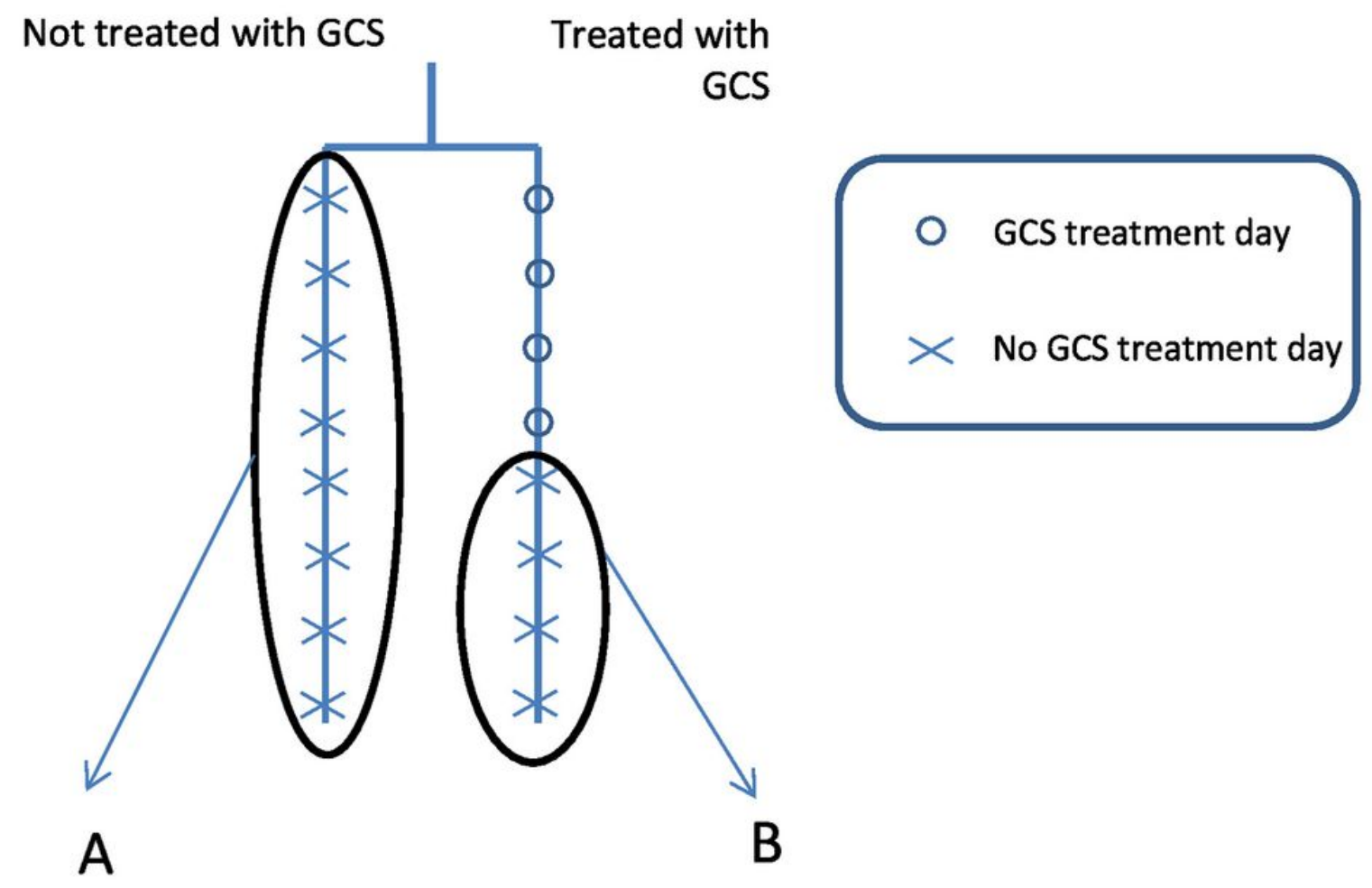

A - All the days of patients who were never treated with GCS.

B - "GCS-free days" of patients who were ever treated with GCS.

Figure 4

Model 4 of the estimated effect of ever being treated with glucocorticoids (GCS) on daily fluid balance $(\mathrm{ml})$ in patients with sepsis in the intensive care unit. This model included only days without GCS treatment. 